\title{
Measuring Qualities of XML Schema Documents
}

\author{
Tin Zar Thaw, Mie Mie Khin \\ Department of Computer Science, University of Computer Studies, Mandalay, Myanmar. \\ Email: tinzar.t@gmail.com
}

Received January $29^{\text {th }}, 2013$; revised March $1^{\text {st }}, 2013$; accepted March $9^{\text {th }}, 2013$

Copyright (C) 2013 Tin Zar Thaw, Mie Mie Khin. This is an open access article distributed under the Creative Commons Attribution License, which permits unrestricted use, distribution, and reproduction in any medium, provided the original work is properly cited.

\begin{abstract}
The Extensible Markup Language (XML) is becoming a de-facto standard for exchanging information among the web applications. Efficient implementation of web application needs to be efficient implementation of XML and XML schema document. The quality of XML document has great impact on the design quality of its schema document. Therefore, the design of XML schema document plays an important role in web engineering process and needs to have many schema qualities: functionality, extensibility, reusability, understandability, maintainability and so on. Three schema metrics: Reusable Quality metric (RQ), Extensible Quality metric (EQ) and Understandable Quality metric (UQ) are proposed to measure the Reusable, Extensible and Understandable of XML schema documents in web engineering process respectively. The base attributes are selected according to XML Quality Assurance Design Guidelines. These metrics are formulated based on Binary Entropy Function and Rank Order Centroid method. To check the validity of the proposed metrics empirically and analytically, the self-organizing feature map (SOM) and Weyuker's 9 properties are used.
\end{abstract}

Keywords: Extensible Markup Language; XML Schema Documents; Web Engineering Process; XML Quality Assurance Design Guidelines; Schema Qualities

\section{Introduction}

Nowadays, the Extensible Markup Language (XML) based web applications are widely used for data exchanging and network services. Efficient implementation of web application needs to be efficient implementation of XML and XML schema document. In web engineering process, metrics are used to measure the quality of the creating software but research of XML schema quality metrics is scare. The proposed metrics are formulated based on the Binary Entropy Function and rank order weight method.

XML Schema has performed two functions: specifying structure relationships of the internal components and specifying mechanisms for validating the content of XML component. Moreover, specifying relationships of XML schema components are very difficult for schema developers if the schema is becoming large. If new schema can be built from previously developed schemas and the schemas are easy to understand and extend for enhancing functions, correcting faults, or adapting them to new circumstances, schemas may save more time than poor quality schemas.

Information theory based software measures are at- tractive because they quantify the physiological complexity of a given Schema document [1]. Binary Entropy Function characterizes the purity of design structure components of a given schema document. The based attributes are identified as having more positive impact on particular qualities of XML schema. There are many rank order weighting methods: the equal weights (EW), rank sum (RS), rank reciprocal (RR), rank-order centroid (ROC) and rank methods. Among these methods, the rank-order centroid (ROC) is a well-known method and produces more accurate result than other methods. The proposed measures are evaluated empirically using the Kohonen Self-Organizing Maps (SOM) algorithm, a non-linear mapping, from a high-dimensional data space to a low-dimensional space. It can cluster efficiently without more understanding the meaning of input data [2].

The usefulness and quality of a new metric is evaluated by using validation process. Theoretical validation is the ensuring process of metrics with the principles of measurement theory and empirical evaluation is the study of software in order to characterize and predict. The two approaches complement one another; a valid set of metrics should be both theoretically validated and empiri- 
cally evaluated. Therefore, a new metric must be evaluated formally and practically for its validation. In order to prove the validation of the presented metric, the metric is evaluated by Nine Weyuker's Properties [3]. The paper is organized as follows. Section 1 introduces about XML and its schema qualities. Section 2 presents the related researches about XML schema metrics. The attribute selection and formulation of proposed metrics are explained in Sections 3 and 4 respectively. The proposed metrics are proved empirically and theoretically in Sections 5 and 6 . Section 7 concludes the paper.

\section{Related Work}

Nowadays, several metrics have been proposed for software developers and software development groups to measure the size and quality of software product during software development process. Many researchers proposed metrics for Document Type Description and Extensible Markup Language (XML) Schema.

The researchers proposed a metric to measure the complexity of Document Type Definition (DTD) documents based on the recursive relationship of elements: the number of elements, connectors, appearance indicators and back edges in a DTD. Their information assisted in comprehending the complexities presented in DTDs and DTD libraries in [4]. Other researchers presented two complexity metrics: Entropy metric and Distinct Structured Element Repetition Scale metric for measuring DTD documents. They compared two metrics with other metrics and they suggested that their metrics were useful in differentiating DTDs with the same size in [5]. Moreover, the most relevant research was done by [6]. In it, a set of five metrics developed for DTD documents to measure the complexity of XML documents and to concentrate on usability and maintainability. They were lines of code, McCabe complexity, structure depth, fan-in and fan-out metrics. They suggested that size and complexity can be applied to complete DTDs.

Bun Yue et al. [7] proposed two composite indices: quality index and complexity index of XML Schema documents focused on the ISO 9126 quality model. The paper [8] proposed a metric to measure the complexity due to the internal architecture and recursion of XML schema components. To validate the metric empirically by comparison with other metrics applied on XML schema documents. They suggested that the more memory and time uses efficiently, the better the schema quality is. The researchers in $[9,10]$ proposed two metrics: Schema Entropy and the total complexity of the XML schema documents to measure the structural complexity of XML schema document. They suggested that their metric provided valuable information about the reliability and maintainability of systems. Visser et al. in [11] proposed a suite of metrics over graph representations of schema structure. These metrics were tree impurity, fan-in, fanout and instability, efferent and afferent coupling and gain instability, coherence and normalized count of modules that were mostly adaptations of existing metrics for other software language. They suggested that the measurement results may be used for assessing potential risks in schemas. According to my knowledge, researchers have been done to determine complexity and maintainable quality of the XML schema documents. There is no the reusable, extensible and understandable qualities measuring. Therefore, in this paper, these quality metrics are proposed for software developers to facilitate private-assessment and improve of their schema based software products in terms of saving cost.

\section{Attribute Selection for XML Qualities}

Selecting the based attributes is great impact on measuring particular software qualities. In this paper, the base attributes are selected according to XML Quality Assurance Design Guidelines.

\subsection{XML Qualities}

There are many qualities for XML Schema documents: understandable, reusable, maintainable, extensible, flexible, compliable and so on [12-16]. Among these qualities, the reusable quality, extensible and understandable quailties are focus on this thesis.

- Reusable: An XM schema component can be globally defined and leveraged by other XML schemas and components in the same document. Reuse concept is that new schema should not be built from scratch, but should be able to leverage previously developed schemas.

- Extensible: Extensible quality $\mathrm{xml}$ schema is possible for developers to write an extension schema by adding additional features to the original in a controlled way.

- Understandable: If the system is difficult to understand for enhancing relationships between schema components or adapting them to new circumstances, changes may increase the development cost. XML schemas are clear, consistent and unambiguous having human readable components.

\subsection{Attribute Selection}

The best practices and the guidelines will allow XML Schema developers to develop good quality schemas. The based attributes are collected according to XML Quality Assurance Design Guidelines [3,4,12,14,16,17]. To get the reusable, extensible and understandable qualities of XML schema documents, many experts guide how attributes are used to get particular quality. 
To achieve a high level of reusable quality, types and elements that are defined globally can be reused in other XML schemas and in the same XML schema documents. If complex types are anonymous, they can't provide reuse property. Inheritance has the property to compartmentalize and reuse the collections of schema elements and attributes by using keywords by extension are used to inheritance schema component structure. For the RQ metric, the following attributes are selected.

- Number of reuse types (Tr).

$$
\begin{aligned}
\operatorname{Tr}= & \text { Number of user defined type declaration } \\
& - \text { Number of anonymous type declaration }
\end{aligned}
$$

- Number of inheritance types(Ti).

$\mathrm{Ti}=$ Number of simple type with restriction

+ Number of complex type with restriction

+ Number of complex type with extension

- Number of global elements (Eg).

- Average number of user defined type references (Mr).

$$
\mathrm{Mr}=\frac{\text { Number of type declaration }}{\text { Number of type definition }}
$$

- Total number of types (Tt).

- Total number of elements (Te).

The inheritance feature type is also support for extensible quality of schema document. For extensible purpose, XML schema send and import types, elements, and attributes from one namespace into the main XML schema by using "include" and "import" tags respectively. The Union types also allow developers to combine types. A wildcard matches element and attribute information items dependent on their namespace name by using the $<$ xs:any $>$ tag to extend schema components. Substitution groups allow elements to be used interchangeable and support the extensible quality of schema document but having many substitution groups tends to more difficult process of these documents. For the extensible quality of XML schema documents, the following based attributes are selected.

- Number of include and import components (I\&I).

- Number of inheritance types (Ti).

- Number of union and any (U\&A).

$$
\begin{array}{r}
U \& A=\text { Number of union components } \\
- \text { Number of any components }
\end{array}
$$

- Number of substitution groups(SG).

- Total Number of Types (Tt).

- Total Number of Elements (Te).

Understandable quality schema directly support for other software qualities: reusable, maintainability, extensible and so on. To get the understandable quality of schema documents, human readable schema components are very important for software developers and develop- ment group. XML Schema provides three understandable components: documentation, annotation and links to some information. Therefore, the UQ metric is proposed on the following based selected metrics.

- Number of annotations (Na).

- Number of documentation (Nd).

- Number of links to requirement and document (Nl).

- Total Number of Nodes (Tn).

\subsection{Assigning Attributes' Weights}

According to guidelines [12-14,16,18], the important of individual attributes are not the same, their weight is not equal. Moreover, many XML schema experts provide the judgments about the positive ranks of attributes for particular quality. Therefore, rank-order centroid (ROC) method is used to calculate weights of attributes for three metrics. In XML schema components, type definition is more important than element declaration. The selected attributes are ranked according to XML schema experts' judgments for each of quality.

For reusable quality, type definitions $\mathrm{Tr}$ and $\mathrm{Ti}$ have higher ranks than other attributes. Eg has the higher reuse than Mr. Tt is used to get the ratios of $\mathrm{Tr}$ and $\mathrm{Ti}$ and Te is used to get the ratios of Eg and Mr. For the RQ metrics, the selected attributes are ranked:

$$
\mathrm{Tr} \geq \mathrm{Ti} \geq \mathrm{Eg} \geq \mathrm{Mr}
$$

According to extensible purpose, I\&I is more important than other internal components. Then type definition, $\mathrm{Ti}$, is ranked. Having many substitution groups tends to more difficult schema process than having many unions. $\mathrm{Tt}$ and Te are used to get the ratios of above attributes. For the EQ metric, the selected attributes are ranked as follows:

$$
\mathrm{I} \& \mathrm{I} \geq \mathrm{Ti} \geq \mathrm{SG} \geq \mathrm{U} \& \mathrm{~A}
$$

For the understandable quality, an annotation component is more important than documentation and link. Many schema documents are more used documentation component than link component. The Tn attribute is used to get the ratio of the ranked attributes. For the UQ metric, the selected attributes are ranked:

$$
\mathrm{Na} \geq \mathrm{Nd} \geq \mathrm{Nl}
$$

Rank-Order Centroid (ROC) method:

$$
\operatorname{ROC}_{i} W(\mu)=\frac{1}{t} \sum_{l=i}^{t} \frac{1}{p_{l}}, i=1,2, \ldots, t
$$

$\mu=$ a metric.

$t=$ the total number of attributes for $\mu$ metric.

$p_{l}=$ the position of $i^{\text {th }}$ attribute for $\mu$ metric.

$$
i \in\left\{\begin{array}{c}
\left\{N_{a}, N_{d}, N_{l}\right\} \mu=U Q \\
\left\{I \& I, T_{i}, S G, U \& A\right\} \mu=E Q \\
\left\{T_{r}, T_{i}, E_{g}, M_{r}\right\} \mu=R Q
\end{array}\right.
$$


Example of Weight Calculation for the based attribute (Tr) of RQ metric:

$$
\begin{aligned}
\operatorname{ROC}_{T r} W(R Q) & =\frac{1}{t} \sum_{l=i}^{t} \frac{1}{p_{l}}, i=1,2, \ldots, t \\
& =\frac{1}{4} *\left(1+\frac{1}{2}+\frac{1}{3}+\frac{1}{4}\right)=0.5208
\end{aligned}
$$

\section{Formulation of Proposed Metrics}

The proposed three metrics are formulated based on the binary entropy Function and rank order centroid method to measure the qualities of XML schema documents. It contains two parts: formulation of proposed metrics and analyzing result of proposed metrics.

\subsection{Formulation of Proposed Metrics}

The proposed three metrics: RQ, EQ and UQ are formulated based on the binary entropy Function and rank order centroid method to measure qualities of a given schema document S. The binary entropy function of based attributes for a given single document is as follow [1]:

$$
\text { Entropy }_{i}(S)=-p_{i^{\oplus}} \log _{2} p_{i^{\oplus}}-p_{i^{\Theta}} \log _{2} p_{i \Theta}
$$

where $p_{i \oplus}$ is the positive concept of $i$ component or a half proportion of positive concept for $i^{\text {th }}$ schema component and $p_{i \Theta \_}$is the negative concept of $i$ component. The positive and negative concepts are given below:

$$
\begin{aligned}
& p_{i^{\oplus}}=\frac{i}{2 D_{i}} \\
& R Q_{i \in\{T r, T i, E g, M r\}}=\left(\sum_{i=1}^{n}\left(\text { Entropy }_{i}(d c . x s d) \times \frac{1}{n} \sum_{l=i}^{n} \frac{1}{p_{l}}\right)\right) \times 100 \\
& =\left(\sum_{i=1}^{n}\left(-p_{i^{\oplus}} \log _{2} p_{i^{\oplus}}-p_{i^{\Theta}} \log _{2} p_{i^{\Theta}} \times \frac{1}{4} \sum_{l=i}^{n} \frac{1}{p_{l}}\right)\right) \times 100 \\
& =\left(-p_{T r^{\oplus}} \log _{2} p_{T r^{\oplus}}-p_{T r^{\Theta}} \log _{2} p_{T r^{\Theta}} \times \frac{1}{4} \sum_{l=1}^{4} \frac{1}{p_{l}}\right) \times 100 \\
& +\left(-p_{T i^{\oplus}} \log _{2} p_{T i^{\oplus}}-p_{T i^{\Theta}} \log _{2} p_{T i \Theta} \times \frac{1}{4} \sum_{l=2}^{4} \frac{1}{p_{l}}\right) \times 100 \\
& +\left(-p_{E g^{\oplus}} \log _{2} p_{E g^{\oplus}}-p_{E g^{\Theta}} \log _{2} p_{E g^{\Theta}} \times \frac{1}{4} \sum_{l=3}^{4} \frac{1}{p_{l}}\right) \times 100 \\
& +\left(-p_{M r^{\Theta}} \log _{2} p_{M r^{\Theta}}-p_{M r^{\Theta}} \log _{2} p_{M r^{\Theta}} \times \frac{1}{4} \sum_{l=4}^{4} \frac{1}{p_{l}}\right) \times 100 \\
& R Q_{i \in\{T r, T i, E g, M r\}}=\left(-\frac{1}{2} \log _{2} \frac{1}{2}-\frac{1}{2} \log _{2} \frac{1}{2}\right) \times 0.5208 \times 100+\left(-\frac{0.5}{2} \log _{2} \frac{0.5}{2}-\frac{1.5}{2} \log _{2} \frac{1.5}{2}\right) \times 0.2708 \times 100 \\
& +\left(-\frac{8}{19} \log _{2} \frac{8}{19}-\frac{11}{19} \log _{2} \frac{11}{19}\right) \times 0.1458 \times 100+\left(-\frac{0.25}{19} \log _{2} \frac{0.25}{19}-\frac{18.75}{19} \log _{2} \frac{18.75}{19}\right) \times 0.0625 \times 100
\end{aligned}
$$

where $D_{i}$ is used to get the ratio concept of $i$ schema component. The $D_{i}$ is defined as follows:

$$
D_{i}=\left\{\begin{array}{c}
T_{t}, i=T_{r} \mid T_{i} \\
T_{n} i=N_{d}\left|N_{l}\right| N_{a} \\
T_{e},(\text { otherwise })
\end{array}\right.
$$

Generally, to measure reusable, extensible and underqualities, the equation is formulated by multiplying with ROC weight method according to Equations (5) and (7):

$$
\mu(S)=\left(\sum_{i=1}^{n} \operatorname{Entropy}_{i}(S) \times R O C_{i} W\right) \times 100
$$

$\mu$ can be RQ, EQ or UQ metric. For each of metrics, the based attributes set according to Equation (6). In all calculation, the entropy value is 1 if the schema contains an equal number of positive and negative components. If To demonstrate three metrics, the xml schema document of dc.xsd is analyzed and measured. This schema document is built as DOM tree and the based attributes are counted as follows.

$$
\{\mathrm{Tr} \rightarrow 2, \mathrm{Ti} \rightarrow 1, \mathrm{Eg} \rightarrow 16, \mathrm{Mr} \rightarrow 0.5\}
$$




$$
R Q i W_{i \in\{T r, T i, E g, M r\}}=88.9978
$$

\subsection{Analyzing Result of Proposed Metrics}

The proposed metrics are analyzed by using $20 \mathrm{XML}$ schema files and the binary entropy function is compared with simple ratio.

\subsubsection{Comparison of Simple Ratio and Binary Entropy Function}

Ratio and Binary Entropy Function Values are between 0 and 1. For example, a given schema document has the total components (20) and Number of Annotation (23). Total component of a give schema document is the sum of total elements, total types and a root component (schema node) of that document. Human understandable components: Number of Annotation, Number of Documentation and Number of links are used to explain types and elements of the same documents. It is clear that if one of human understandable components is larger than the total components for a given schema document, the result can be less than one. To solve this problem, in simple type ratio, the ratio is $23 / 20(1.15)$ and it is greater than one. Binary Entropy function can solve this problem as follows:

$$
\text { Entropy }_{i}(S)=-p_{i^{\oplus}} \log _{2} p_{i^{\oplus}}-p_{i^{\Theta}} \log _{2} p_{i^{\Theta}}
$$

$$
\begin{aligned}
& \text { Entropy }{ }_{\mathrm{Na}}(\text { ExampleSchema }) \\
& =-p_{\mathrm{Na}^{\oplus}} \log _{2} p_{\mathrm{Na}^{\oplus}}-p_{\mathrm{Na}^{\oplus}} \log _{2} p_{\mathrm{Na}^{\oplus}}-p_{\mathrm{Na}^{\Theta}} \log _{2} p_{\mathrm{Na}^{\Theta}} \\
& =-\frac{11.5}{20} \log _{2} \frac{11.5}{20}-\frac{8.5}{20} \log _{2} \frac{8.5}{20} \\
& =0.983708
\end{aligned}
$$

The binary entropy function value is not exceeded one as well as can reduce the human readable value having more than total components. Therefore, the proposed metrics is formulated by summarization the binary entropy functions of based metrics and multiplying their rank weights.

\subsubsection{Analyzing Result of Proposed Metrics}

To analyze the proposed metrics, the attributes and three qualities of the following schema documents are analyzed and calculated. These schema document links are shown in Table 1 in Appendix.

The correlation between reusable related attributes and the RQ metric value for reusable quality of XML schema documents are analyzed in Figure 1. There are the ratios of $\mathrm{Tr}$ and $\mathrm{Ti}$ to $\mathrm{Tt}$ and ratios of $\mathrm{Eg}$ and $\mathrm{Mr}$ to $\mathrm{Te}$. In this analysis, the schemas: ID_34 has the highest value at 77.22 because the ratios of $\mathrm{Tr}$ and $\mathrm{Ti}$ (two third and one third of Tt), the ratio of Eg (half a Te), the ratio of $\operatorname{Mr}(23$ times smaller than Te) . For the schema: ID_17, Te is the

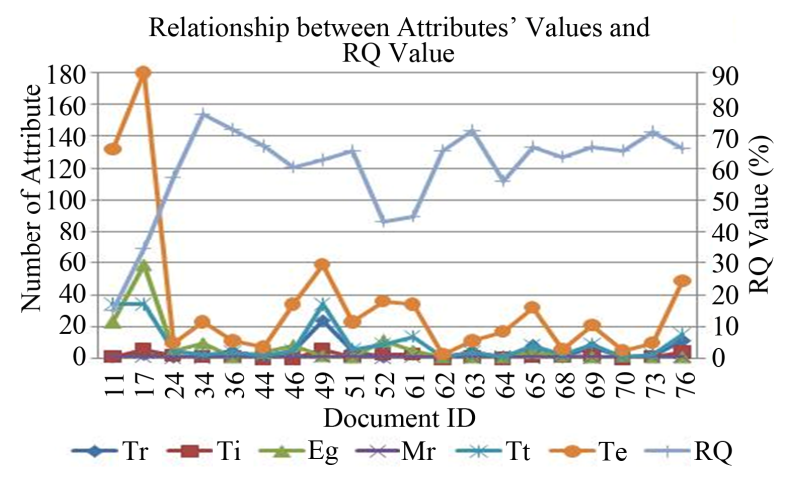

Figure 1. Reusable related attributes' values vs RQ metric value.

highest number but the Eg value is less than one third of $\mathrm{Te}$ and $\mathrm{Mr}$ is very low value. Moreover, $\mathrm{Tr}$ is eleven times smaller than $\mathrm{Tt}$ and $\mathrm{Ti}$ is seven times smaller. Therefore, the schema: ID_17 has lower reusable quality value than ID_34.

The correlation between extensible related attributes and the EQ metric value for extensible quality of XML schema documents are analyzed in Figure 2.

There are the I\&I, U\&A and SG ratios to Te and the Ti ratio to Tt. In this analysis, the XML schema document: ID_34 has higher value than other schema documents because of the overall highest ratio.

The correlation between human readable attributes and the UQ metric value for understandable quality of XML schema documents are analyzed in Figure 3. There are the ratios of $\mathrm{Na}$, $\mathrm{Nd}$ and $\mathrm{Nl}$ to $\mathrm{Tn}$. In this analysis, it is clear that higher human readable attributes' ratios tend to higher the understandable quality of XML schema document.

\section{Empirical Validation of Proposed Metrics}

To prove the validity and usefulness of the proposed metrics, the clustering method, Self-organizing maps (SOM) and well-known XML schemas are used. One hundred XML schemas are downloaded for validating the proposed metrics that are shown in Table 1. These schemas are: WSDL Schemas for describing network services, Mathematical Schemas for calculation, Metadata Vocabulary Schemas for monitoring and changing systems affecting everything in life and Geospatial Schemas for developers to ensure complex spatial information. XML schema documents are clustered by SOM depending on two types: only particular proposed metric and it's based attributes and the cluster matching results are compared. It is clear that the proposed metrics is validated and useful according to the SOM clustering result.

\subsection{Self-Organizing Map Clustering}

The unsupervised learning method, SOM is suitable for 


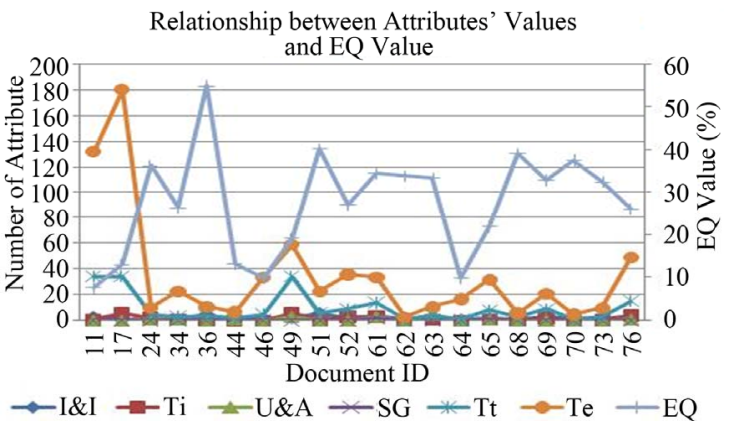

Figure 2. Reusable related attributes' values vs EQ metric value.

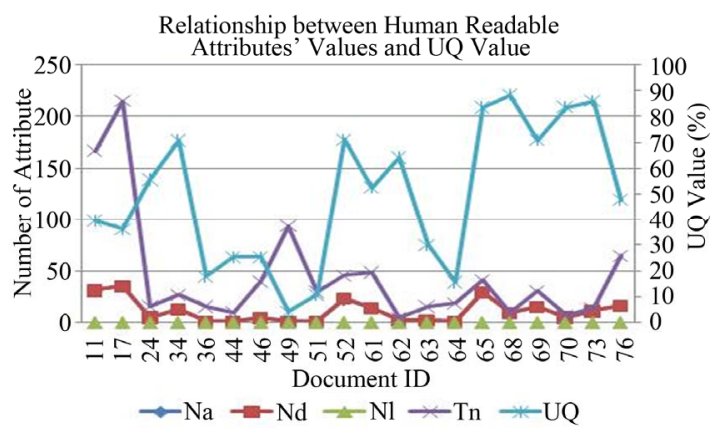

Figure 3. Human readable attributes' values vs UQ metric value.

measuring the validity and usefulness of the proposed metrics that they are difficult to prove their validity and usefulness because they don't have historical data and other comparison metrics. One advantage is that they do not require more understanding with input data. The SOM learns similarity of input feature vectors and responds groups of similar input vectors [2].

Before clustering XML schema documents, two data files are created for each of proposed metrics, one contains the particular proposed metric values of one hundred XML schema documents and another contains its based attributes of those. In preprocessing step, file attributes are converted into binary data according to Equations (12) and (13).

$$
f(x)=\left\{\begin{array}{l}
1, \text { if }\left(x>\frac{f(y)}{2}\right) \\
0, \text { (otherwise) }
\end{array}\right.
$$

where $f(x)$ : the binary conversion function that converts the $x$ based metric value into binary data and $f(y)$ : the function that chooses $T_{t}, T_{e}, T_{n}$ and $\operatorname{Max}\left(T_{n}\right)$ based on $y$.

$$
f(y)=\left\{\begin{array}{l}
T_{t}, \text { else if }(y=t r \mid t i) \\
T_{e}, \text { else if }(y=E g|M r| U \& A \mid S G) \\
T_{n}, \text { else if }(y=T t|T e| N a|N d| N l \mid I \& I) \\
M A X\left(T_{n} \text { attributes }\right) \text { otherwise }
\end{array}\right.
$$

After converting binary data, the contingency tables are created for each of six files. For creating the contingency table, there are the variables $a, b, c$ and $\mathrm{d}, i^{\text {th }}$ position, the attribute $x$ of a record and the attribute $y$ of another record.

To create the contingency table, dissimilarities are calculated by Jaccard coefficient [6]:

$$
d(x, y)=\frac{e}{e+f+g}
$$

$e=$ number of times $x_{i}=1$ and $y_{i}=1$.

$f=$ number of times $x_{i}=0$ and $y_{i}=1$.

$g=$ number of times $x_{i}=1$ and $y_{i}=0$.

These dissimilarity matrixes of schema files are trained with the SOM and the schema documents of each file are cluster in terms of dissimilarity matrixes. For instance, the two files of EMC metric are clustered by SOM and the cluster matching result is analyzed.

SOM clusters XML schema documents into two groups depending on the based attributes $\mathrm{Na}, \mathrm{Nd}$ and $\mathrm{Nl}$. The first group contains schema IDs: 65, 68, 70, 73, 96 and 99 and the second group contains other schema documents in Figure 4. Moreover, in Figure 5, SOM also clusters XML schema documents into two groups depending on only the UQ metric values. The first group contains schema IDs: 24, 34, 52, 61, 62, 65, 68, 69, 70 and 73 and the second group, other group contains other schema documents. The first groups' members of two

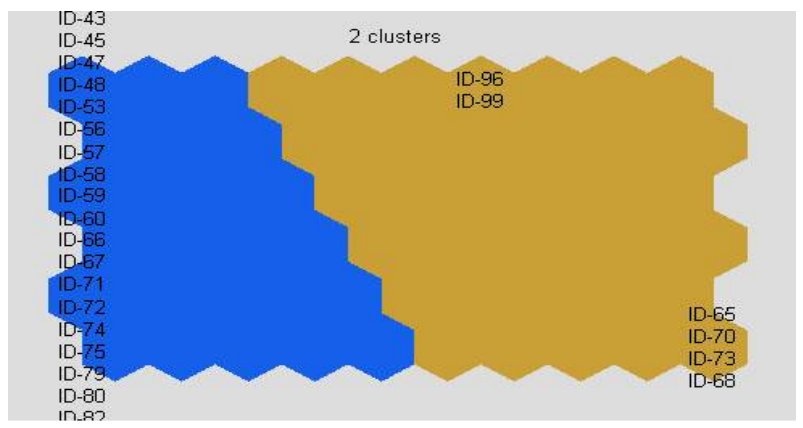

Figure 4. SOM clustering result depending on based attributes.

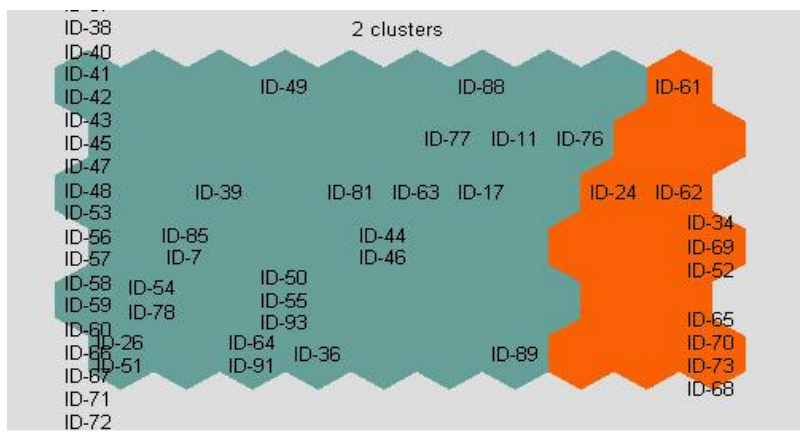

Figure 5. SOM clustering result depending on only proposed UQ metric. 
types are then compared and analyzed. It is clear that four schema documents are matched and eight schema documents are not equal. Moreover, the second groups' members of two types are also compared and analyzed. The result shows that 82 schema documents are matched. Therefore the total cluster matching result is $82 \%$.

For all proposed metrics, the cluster matching results are greater than 80 percent. Therefore, the proposed three metrics are useful to measure reusable, extensible and understandable qualities of XML schema documents.

\subsection{Measuring and Evaluation of Proposed Metrics}

To prove the validity and usefulness of the proposed RQ, EQ and UQ metrics, a hundred of standard XML schemas are used. To analyze the SOM cluster matching results, the cluster thresholds are assumed and are compared with SOM cluster thresholds of two types for each of four proposed metrics. The SOM clusters XML schema documents according to two types: depending on only the proposed metric and depending on the based attributes of the proposed metric and produces two groups for each of types. One group of a type is then compared with one group of other type and the cluster matching results of two types is produced.

According to Figure 6, 66 schema documents do not contained human readable components and therefore their understandable quality are zero. The SOM can produce these documents into one group in terms of only UQ value. But, the SOM cannot produce them into one group in term of based attributes and 2 documents contain in another group. It is clear that the SOM clustering result depending on only proposed UQ metric can produce more accurate result than depending on the based attributes of UQ metric. Moreover, the two SOM clustering results are compared with the assumed clustering result, the cluster matching results are $100 \%$ in terms of only UQ value and $92 \%$ in terms of the based metrics of UQ.

To analyze the clustering results of two types, 100 $\mathrm{XML}$ schema documents are assumed into two groups:

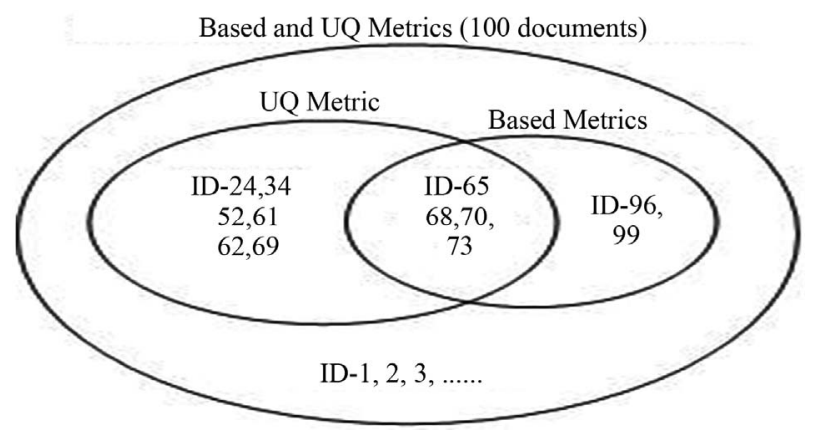

Figure 6. Cluster matching of UQ and its based metrics. from 7.8981 to 17.165 (13 schema documents) and from 17.165 to 77.124 (87 schema documents). The SOM clustering results of RQ metric are shown in Figure 7. Tr, Ti, Eg, Mr, Tt and Te are the based attribute of the RQ metric. The two SOM clustering results are compared with the assumed clustering result, the cluster matching results are $100 \%$ in terms of only RQ value and $85 \%$ in terms of the based metrics of RQ.

SOM clustering results of EQ metric are shown in Figure 8. I \& I, Ti, SG, U \& A, Tt and Te are the based attributes of the EQ metric. The two SOM clustering results are compared with the assumed clustering result, the cluster matching results are $100 \%$ in terms of only EQ value and $96 \%$ in terms of the based metrics of EQ.

According to the analyzed result empirically, the thee metrics are more suitable for measuring reusable, extensible and understandable qualities of XML schema documents than the based attributes because of matching result with more than $80 \%$.

\section{Analytical Validation of the Proposed Metrics}

The usefulness and quality of a new metric is evaluated by using validation process analytically. To validate the proposed three metrics, the well known Weyuker's properties [11] are used.

\section{Analytical Evaluation of the Proposed Metrics against Weyuker's Properties}

In this section, the validation of the proposed metrics is

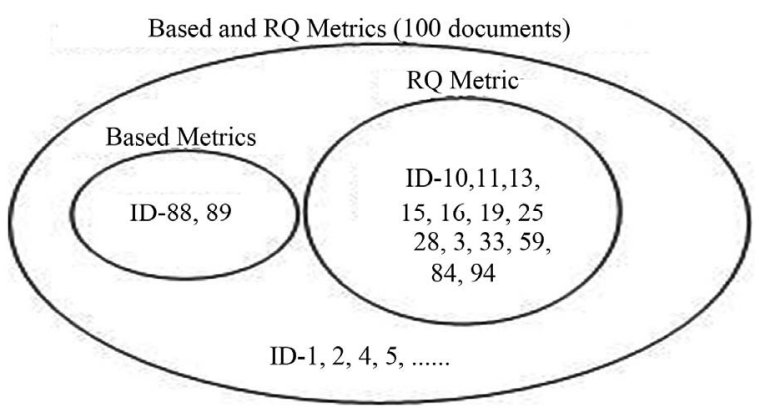

Figure 7. Cluster matching of $R Q$ and its based metrics.

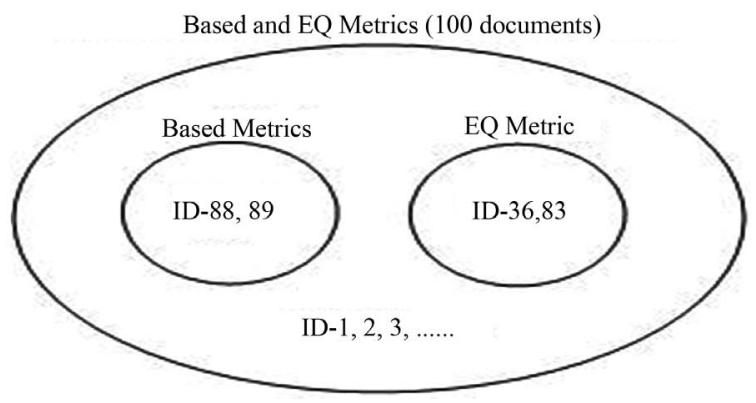

Figure 8. Cluster matching of EQ and its based metrics. 
also proved by using nine well known Weyuker's properties through examples [12]. To validate the analytical validation, assume a metric $(\mu)$, three distinct schema documents (A, B and $\mathrm{C}$ ) and the combined $\mathrm{AB}, \mathrm{AC}$ and $\mathrm{BC}$ documents. For instance, the $\mathrm{AB}$ documents is combined the A document with the $\mathrm{B}$ document.

The evaluations of the proposed metric against Weyuker's properties are as follows.

1) Non-Coarseness $((\exists A)(\exists B)(\mu(A) \neq \mu(B)))$ : This means that a metric has not to measure and produce the same value for all schema documents. It is clear that three proposed metrics satisfy this property.

2) Granularity: Let $c$ be a non-negative number, and then there are only finite numbers of schema documents of complexity $\boldsymbol{c}$. This states that there are only a finite number of XML schema documents of the same complexity. The proposed metrics satisfy this property because of the same complexity values with the same ratios having the same rank.

3) Non-uniqueness $(\boldsymbol{\mu}(\mathbf{A})=\boldsymbol{\mu}(\mathrm{B}))$ : This means that there are distinct schema documents of the same complexity value. For example, in the Table 1, the schema A (ID_64) is metadata vocabulary schema and the schema B (ID_46) is a geospatial schema for developer to develop publicly available interface standards for spatial information. The EQ metric will be able to demonstrate the equalities of schema document in terms of their corresponding attributes' ratios. Therefore the proposed metrics satisfy this property.

4) Design Details are important $(\mu(\mathrm{A}) \equiv \mu(\mathrm{B})$ and $\mu(\mathrm{A})$ $\neq \boldsymbol{\mu}(\mathbf{B}))$ : This property means that the details of the structural design can change the metric value having the same functions. In Figure 9, A and B are the different schema documents having the same functionality, their attributes' values can be different because of different implementation. Therefore, $\operatorname{Tr}$ value is 0 because of the anonymous type in the schema $\mathrm{A}$. In the schema $B$, Tr value is 1 because the type can be reused in the same schema or other schemas.

5) Monotonic Complexity $(\mu(\mathrm{A}) \leq \mu(\mathrm{AB})$ and $\mu(\mathrm{B}) \leq$ $\boldsymbol{\mu}$ (AB)): This means that the metric value of the combination schema can never be less than individual schema values. For example, ID_46 and ID 64 have the same EQ values and their combination schema value is equal individual schema values. The pro-

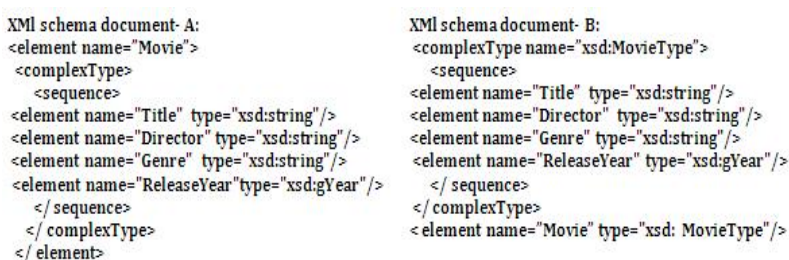

Figure 9. Listing of structural design A and design B. posed metrics satisfy this property because the combination schema has the same value.

6) Non-equivalence of Interaction $(\mu(\mathrm{A})=\mu(\mathrm{B})$ and $\mu$ $(\mathbf{A C})=\boldsymbol{\mu}(\mathbf{B C}))$ : This states that even thought $\mathrm{A}$ and $\mathrm{B}$ has the same values, interaction between $\mathrm{A}$ and $\mathrm{C}$ and between $\mathrm{B}$ and $\mathrm{C}$ can be different resulting in different quality values. For example, ID_46 and ID_64 are the schema A and B. ID_60 lets the schema $\mathrm{C}$ and has different extensible quality in Table 1 . The proposed metrics satisfy this property because the AC and BC schemas have different the EQ values with 20.3768 and 17.91042 respectively.

7) Permutation Changes Complexity: This property means that permutation impact on the metric value. The proposed metrics satisfy this property according to Figure 9.

8) Renaming Property: This property states that when the name of the measured schema component changes, the metric value can't be changed. The proposed metrics satisfy this property.

9) Interaction Increases Complexity $(\mu(A)+\mu(B) \leq \mu$ (AB)): This property is that the interaction between two schemas cannot decrease their combination metric value. This property need not necessarily be true because of the percentage quality value of XML schema documents.

According to analytical analyzed result, the proposed metrics are robust with satisfying eight Weyuker's properties.

\section{Conclusion}

Three quality metrics: RQ, EQ and UQ are proposed to measure the reusable, extensible and understandable qualities of XML Schema documents based on binary entropy function and rank order centroid method respecttively. The SOM method is used to cluster the XML schema documents to validate of the proposed three metrics empirically. To compare the matching clustering results of two types: depending on only one of each proposed metrics and depending on its based attributes are greater than $80 \%$. According to the analyzed results empirically, the proposed metrics are more suitable to measure the three qualities than their based attributes. The metrics are evaluated by using Weyuker's 9 properties analytically. The RQ, EQ and UQ metrics are robust satisfying with eight properties. Therefore, the proposed metrics can provide valuable information for improving the quality of XML based system.

\section{Acknowledgements}

This work supported by University of Computer Studies, Mandalay, Myanmar. All teachers taught about this work 
and all teachers in my life are gracefully acknowledged.

\section{REFERENCES}

[1] T. M. Cover and J. A. Thomas, "Elements of Information Theory," 2nd Edition, John Wiley \& Sons, Inc., Hohoken, New Jersey and Published by Simultaneously in, 2006.

[2] F. Lourenco, V. Lobo and F. Bacao, "Binary Based Similarity Measures for Categorical Data and Their Applications in Self-Organizing Maps," JOCLAD 2004-XI Jornadas de Classificacao e Anlise de Dados, Lisbon, 1-3 April 2004, pp. 1-8.

[3] E. J. Weyuker, "Evaluation Software Complexity Measures," IEEE Transactions on Software Engineering, Vol. 14, No. 9, 1988, pp. 1357-1365. doi:10.1109/32.6178

[4] Y. Chen and R. McFadyen, "A DTD Complexity Metric,” Proceedings of the 21st IASTED International Conference, Applied Informatics, Innsbruck, 10-13 February 2001, Austria, pp. 1045-1052.

[5] S. Misra and B. Dilek, "Document Type Definition (DTD) Metrics," Romanian Journal of Information, Science and Technology, Vol. 14, No. 1, 2011, pp. 31-50.

[6] M. Klettke, L. Schneider and A. Heuer, "Metrics for XML Document Collections," Lecture Notes in Computer Science, Vol. 2490, 2002, pp. 15-28.

[7] A. McDowell, C. Schmidt and K. Bun Yue, “Analysis and Metrics of XML Schema," Proceedings of International Conference on Software Engineering Research and Practice, 2004, pp. 538-544.

[8] D. Basci and S. Misra, "Measuring and Evaluating a Design Complexity Metric for XML Schema Documents," Journal of Information Science and Engineering, Vol. 25, No. 5, 2009, pp. 1405-1425.

[9] D. Basci and S. Misra, "Entropy as a Measure of Quality of XML Schema Document,” International Arab Journal of Information Technology, Vol. 8, No. 1, 2010, pp. 75-
83.

[10] D. Basci and S. Misra, "Complexity Metric for XML Schema Documents," Proceedings of the 5th International Workshop on SOA and Web Practices, 2007, pp. $1-14$.

[11] J. Visser, "Structure Metrics for XML Schema," Proceedings of XATA, Portugal, 2006, pp. 1-12.

[12] B. Sumak, M. Hericko and M. Pusnik, "Towards a Framework for Quality XML Schema Evaluation,” Proceedings of the ITI 2007 29th International Conference on Information Technology Interfaces, Cavtat, 25-28 June 2007, pp. 783-788.

[13] D. Obasanjo, Microsoft Corporation, "W3C XML Schema Design Patterns: Avoiding Complexity,” Applies to: W3C XML Schema, 2003. http://www.xml.com

[14] H. S. Thompson, D. Beech, M. Maloney and N. Mendelsohn, "XML Schema Part 1: Structures Second Edition," W3C Recommendation, 2004. http://www.w3.org/TR/xmlschema-1/

[15] D. Stephenson, “XML Schema Best Practice,” HewlettPackard Development Company, Palo Alto, 2004.

[16] "PESC Guidelines for XML Architecture and Data Modeling," a Publication of the Postsecondary Electronic Standards Council (PESC), Version 3.0, 2005.

[17] T. Z. Thaw, "Measuring and Evaluation of Reusable Quality and Extensible Quality for XML Schema Documents," 2011 IEEE Student Conference on Research and Development, Cyberjaya, 19-20 December 2011, pp. 473478.

[18] D. Lee and W. W. Chu, "Comparative Analysis of Six XML Schema Languages,” ACM SIGMOD Record, Vol. 29, No. 3, 2000, pp. 76-87. doi:10.1145/362084.362140 


\section{Appendix}

Table 1. The proposed metrics' values of XML schema document.

\begin{tabular}{|c|c|c|c|c|}
\hline Document -ID & Schema Document Links & RQ & EQ & UQ \\
\hline 1 & http://www.multispeak.org/interface/30j/10_OA_EA.asmx?WSDL & 55.615 & 5.7096 & 0 \\
\hline 3 & http://services.argosoft.com/AddressValidation/AddressVerifier.asmx?WSDL & 9.6491 & 0 & 0 \\
\hline 4 & https://api.channeladvisor.com/ChannelAdvisorAPI/v5/AdminService.asmx?WSDL & 56.214 & 6.369 & 0 \\
\hline 5 & http://b3.caspio.com/ws/api.asmx?wsdl & 65.717 & 21.981 & 0 \\
\hline 6 & http://www.oorsprong.org/websamples.arendsoog/ArendsoogbooksService.wso?WSDL & 35.742 & 0 & 0 \\
\hline 7 & http://www.w3.org/Math/XMLSchema/mathml2/content/arith.xsd & 68.793 & 0 & 7.5553 \\
\hline 8 & http://www.yazgelistir.com/YGServices/ArticleService.asmx?wsdl & 28.313 & 1.79 & 0 \\
\hline 10 & http://services.nirvanix.com/ws/Authentication.asmx?WSDL & 8.6739 & 0 & 0 \\
\hline 11 & http://schemas.opengis.net/sensorML/1.0.1/base.xsd & 15.195 & 7.6663 & 39.6267 \\
\hline 12 & http://banguat.gob.gt/variables/ws/BDEF.asmx?WSDL & 46.649 & 0 & 0 \\
\hline 13 & http://www.webservicex.net/BibleWebservice.asmx?wsdl & 10.309 & 0 & 0 \\
\hline 14 & http://www.thomas-bayer.com/axis2/services/BLZService?wsdl & 60.155 & 0 & 0 \\
\hline 15 & http://www.mathertel.de/AJAXEngine/S02_AJAXCoreSamples/CalcService.asmx?WSDL & 10.474 & 0 & 0 \\
\hline 16 & http://ww2.wso2.org/ charitha/calculator.xsd & 9.6411 & 0 & 0 \\
\hline 17 & http://schemas.opengis.net/wms/1.3.0/capabilities_1_3_0.xsd & 34.822 & 13.096 & 36.1851 \\
\hline 18 & http://ssl.9squared.com/catalog/catalog.asmx?WSDL & 50.274 & 2.6522 & 0 \\
\hline 20 & http://service.ecocoma.com/convert/chinese.asmx?WSDL & 24.61 & 0 & 0 \\
\hline 21 & http://service.ecocoma.com/geo/cityzip.asmx?WSDL & 52.367 & 0 & 0 \\
\hline 22 & http://schemas.opengis.net/context/1.1.0/collection.xsd & 55.225 & 26.21 & 0 \\
\hline 23 & http://svc.exaphoto.com/eXaPhoto/CollectionServices.asmx?WSDL & 54.21 & 6.9871 & 0 \\
\hline 24 & http://www.w3.org/Math/XMLSchema/mathml2/presentation/common.xsd & 57.318 & 36.255 & 55.5796 \\
\hline 25 & http://quiksilver.ws.eto.fr/Connexion.asmx?WSDL & 9.1359 & 0 & 0 \\
\hline 26 & http://www.w3.org/Math/XMLSchema/mathml2/content/constants.xsd & 68.707 & 0 & 10.069 \\
\hline 27 & http://www.esendex.com/secure/messenger/soap/ContactService.asmx?WSDL & 37.636 & 4.5673 & 0 \\
\hline 28 & http://api.legiomedia.com/Content.asmx?WSDL & 7.8981 & 2.0172 & 0 \\
\hline 29 & http://www.webservicex.net/ConverPower.asmx?WSDL & 63.314 & 17.603 & 0 \\
\hline 30 & http://www.webservicex.net/ConvertTemperature.asmx?WSDL & 63.314 & 17.603 & 0 \\
\hline 31 & http://www.webservicex.net/CovertPressure.asmx?WSDL & 63.314 & 17.603 & 0 \\
\hline 32 & https://demo.docusign.net/API/3.0/Credential.asmx?WSDL & 32.316 & 6.0195 & 0 \\
\hline 33 & http://www.webservicex.net/CreditCard.asmx?WSDL & 12.259 & 0 & 0 \\
\hline 34 & http://ns.nsdl.org/schemas/MRingest/crsd_v1.06.xsd & 77.124 & 26.416 & 70.7804 \\
\hline 35 & http://www.webservicex.com/CurrencyConvertor.asmx?wsdl & 64.193 & 17.603 & 0 \\
\hline
\end{tabular}




\section{Continued}

\begin{tabular}{|c|c|c|c|c|}
\hline 36 & http://schemas.opengis.net/sld/1.1.0/DescribeLayer.xsd & 72.356 & 54.989 & 17.8333 \\
\hline 37 & http://ws.interfax.net/dfs.asmx?WSDL & 40.329 & 4.5992 & 0 \\
\hline 38 & http://service.ecocoma.com/geo/distance.asmx?WSDL & 31.961 & 0 & 0 \\
\hline 39 & http://www.w3.org/Math/XMLSchema/mathml2/content/elementary-functions.xsd & 67.026 & 0 & 6.0966 \\
\hline 40 & http://ws2.serviceobjects.net/ev/EmailValidate.asmx?WSDL & 33.48 & 0 & 0 \\
\hline 41 & http://ws.cdyne.com/emailverify/Emailvernotestemail.asmx?wsdl & 34.065 & 0 & 0 \\
\hline 42 & http://rangiroa.essi.fr:8080/dotnet/evaluation-cours/EvaluationWS.asmx?WSDL & 26.43 & 0 & 0 \\
\hline 43 & http://schemas.opengis.net/wms/1.3.0/exceptions_1_3_0.xsd & 75.543 & 21.969 & 0 \\
\hline 44 & http://schemas.opengis.net/filter/2.0/expr.xsd & 66.985 & 13.249 & 25.4578 \\
\hline 45 & http://developer.factiva.com/2.0/wsdl/FDKParsers.wsdl & 58.304 & 10.79 & 0 \\
\hline 46 & http://schemas.opengis.net/se/1.1.0/FeatureStyle.xsd & 60.39 & 9.9698 & 25.4578 \\
\hline 47 & http://demo.soapam.com/services/FedEpayDirectory/FedEpayDirectoryService?WSDL & 39.527 & 0 & 0 \\
\hline 48 & http://service.ecocoma.com/shipping/fedex.asmx?WSDL & 46.052 & 0 & 0 \\
\hline 49 & http://schemas.opengis.net/filter/2.0/filterCapabilities.xsd & 62.678 & 19.183 & 4.2523 \\
\hline 50 & http://www.w3.org/Math/XMLSchema/mathml2/content/functions.xsd & 66.079 & 0 & 17.0165 \\
\hline 51 & http://schemas.opengis.net/sld/1.1.0/GetMap.xsd & 65.385 & 40.3 & 10.8705 \\
\hline 52 & http://ns.nsdl.org/schemas/MRingest/harvest_v1.01.xsd.sav & 43.316 & 27.14 & 71.0337 \\
\hline 53 & http://terraserver-usa.com/LandmarkService.asmx?WSDL & 58.767 & 8.7403 & 0 \\
\hline 54 & http://www.w3.org/Math/XMLSchema/mathml2/content/linear-algebra.xsd & 62.389 & 0 & 9.1801 \\
\hline 55 & http://www.w3.org/Math/XMLSchema/mathml2/content/logic.xsd & 68.02 & 0 & 17.0165 \\
\hline 56 & http://www.chemspider.com/MassSpecAPI.asmx?WSDL & 33.968 & 3.4028 & 0 \\
\hline 57 & http://www.w3.org/Math/XMLSchema/mathml3/mathml3-common.xsd & 61.058 & 20.35 & 0 \\
\hline 58 & http://www.w3.org/Math/XMLSchema/mathml3/mathml3-strict-content.xsd & 44.91 & 19.126 & 0 \\
\hline 59 & http://hooch.cis.gsu.edu/bgates/MathStuff/Mathservice.asmx?WSDL & 8.9668 & 0 & 0 \\
\hline 60 & http://demo.turtletech.com/latest/webAPI/metering.asmx?WSDL & 46.47 & 3.8511 & 0 \\
\hline 61 & http://schemas.opengis.net/sensorML/1.0.1/method.xsd & 45.103 & 34.533 & 52.5938 \\
\hline 62 & http://www.exchangenetwork.net/repository/schema/NetDMR/1/0/NetDMR_Permits_v1.0.xsd & 65.62 & 33.853 & 64.1722 \\
\hline 63 & http://ns.nsdl.org/schemas/nsdl_search/nsdl_search_v1.02.xsd & 72.03 & 33.446 & 29.9817 \\
\hline 64 & http://www.openarchives.org/OAI/2.0/oai_dc.xsd & 56.068 & 9.9698 & 15.606 \\
\hline 65 & http://schemas.opengis.net/om/1.0.0/observation.xsd & 66.783 & 22.001 & 83.316 \\
\hline 66 & https://www.devcallnow.com/WebService/OneCallNow.asmx?wsdl & 28.053 & 5.6442 & 0 \\
\hline 67 & https://api.channeladvisor.com/ChannelAdvisorAPI/v3/OrderService.asmx?WSDL & 70.996 & 18.636 & 0 \\
\hline 68 & http://schemas.opengis.net/ows/2.0/owsExceptionReport.xsd & 63.52 & 39.154 & 88.2477 \\
\hline 69 & http://schemas.opengis.net/ows/2.0/owsGetCapabilities.xsd & 66.939 & 32.962 & 70.9559 \\
\hline 70 & http://schemas.opengis.net/ows/2.0/owsGetResourceByID.xsd & 65.537 & 37.598 & 83.582 \\
\hline 71 & http://trial.serviceobjects.com/pa/phoneappend.asmx?WSDL & 45.482 & 0 & 0 \\
\hline 72 & http://service.thefamousgroup.com/ProjectService.asmx?wsdl & 57.959 & 6.1383 & 0 \\
\hline
\end{tabular}




\section{Continued}

\begin{tabular}{|c|c|c|c|c|}
\hline 73 & http://ns.nsdl.org/schemas/provenance_about/provenance_about_v1.01.xsd & 71.393 & 32.518 & 85.9227 \\
\hline 74 & http://www.partenairedejeu.fr/WebServices/RelationManager.asmx?WSDL & 43.209 & 8.9402 & 0 \\
\hline 75 & http://www.hitslink.com/reportws.asmx?WSDL & 66.304 & 14.151 & 0 \\
\hline 76 & http://ns.nsdl.org/schemas/ndr/response_v1.00.xsd & 66.537 & 26.138 & 47.8354 \\
\hline 77 & http://www.oasis-open.org/committees/regrep/documents/2.0/schema/rs.xsd & 30.297 & 18.692 & 34.95 \\
\hline 78 & http://www.w3.org/Math/XMLSchema/mathml2/presentation/scripts.xsd & 62.389 & 0 & 9.1801 \\
\hline 79 & http://quisque.com/fr/chasses/blasons/search.asmx?WSDL & 42.766 & 0 & 0 \\
\hline 80 & http://www.phdcc.com/findinsite/SearchService.asmx?wsdl & 54.41 & 5.7096 & 0 \\
\hline 81 & http://www.w3.org/Math/XMLSchema/mathml2/content/semantics.xsd & 64.339 & 2.1081 & 22.212 \\
\hline 82 & http://gw1.aql.com/soap/sendsmsservice.php?wsdl & 67.379 & 18.206 & 0 \\
\hline 83 & https://portal.bmi.gv.at/ref/wsdl/zmr/test/wsdl/Service.wsdl & 72.613 & 63.494 & 0 \\
\hline 84 & http://www.sipeaa.it/wset/ServiceET.asmx?WSDL & 15.503 & 0 & 0 \\
\hline 85 & http://www.w3.org/Math/XMLSchema/mathml2/content/sets.xsd & 68.004 & 6.0195 & 6.7416 \\
\hline 86 & http://www.geoservicios.com/V2.0/sgeo/sgeo.asmx?WSDL & 44.88 & 0 & 0 \\
\hline 87 & http://e-commerce.pvc.maricopa.edu/cis234/old/ssilkey/SimpleAddress.xsd & 60.447 & 24.868 & 0 \\
\hline 88 & http://www.w3.org/Math/XMLSchema/mathml2/presentation/space.xsd & 68.355 & 22.673 & 36.7842 \\
\hline 89 & http://www.w3.org/Math/XMLSchema/mathml2/presentation/style.xsd & 59.15 & 5.0705 & 41.689 \\
\hline 90 & http://msrmaps.com/TerraService2.asmx?WSDL & 46.85 & 5.2641 & 0 \\
\hline 91 & http://www.w3.org/Math/XMLSchema/mathml2/content/tokens.xsd & 76.467 & 17.603 & 15.606 \\
\hline 92 & http://www.webservicex.net/TranslateService.asmx?wsdl & 63.715 & 17.603 & 0 \\
\hline 93 & http://www.w3.org/Math/XMLSchema/mathml2/content/vector-calculus.xsd & 63.119 & 0 & 17.0165 \\
\hline 94 & http://services.test.musiccue.net/rapidcueapplication/WorkManager.asmx?WSDL & 9.7771 & 0 & 0 \\
\hline 95 & http://www.imagine-r.com/services/WsImagineR.asmx?WSDL & 46.628 & 2.7369 & 0 \\
\hline 96 & http://www.xignite.com/xMetals.asmx?WSDL & 58.867 & 16.564 & 0 \\
\hline 97 & http://www.xignite.com/xNASDAQLastSale.asmx?WSDL & 62.755 & 18.449 & 0 \\
\hline 98 & http://www.xignite.com/xNews.asmx?WSDL & 56.067 & 11.206 & 0 \\
\hline 99 & http://www.xignite.com/xQuotes.asmx?WSDL & 58.823 & 14.558 & 0 \\
\hline 100 & http://www.xignite.com/xwatchlists.asmx?WSDL & 61.415 & 16.265 & 0 \\
\hline
\end{tabular}

